\begin{tabular}{|c|c|}
\hline Title & $\begin{array}{l}\text { Passive Shimming by Eliminating Spherical Harmonics Coefficients of all Magnetic Field Components Generated by } \\
\text { Correction Iron Pieces }\end{array}$ \\
\hline Author(s) & Noguchi, So; Kim, SeokBeom; Hahn, Seungyong; I wasa, Y ukikazu \\
\hline Citation & $\begin{array}{l}\text { IEEE Transactions on Magnetics, 50(2), } 7014904 \\
\text { https://doi.org/10.1109/ MA G.2013.2276736 }\end{array}$ \\
\hline Issue Date & 201402 \\
\hline Doc URL & http:/hdl.handle.net/2115/56401 \\
\hline Rights & $\begin{array}{l}\text { C } 2014 \text { IEEE. Personal use of this material is permitted. Permission from IEEE must be obtained for all other uses, in } \\
\text { any current or future media, including reprinting/republishing this material for advertising or promotional purposes, } \\
\text { creating new collective works, for resale or redistribution to servers or lists, or reuse of any copyrighted component of } \\
\text { this work in other works. }\end{array}$ \\
\hline Tyре & article (author version) \\
\hline File Information & MAS_revised.pdf \\
\hline
\end{tabular}

Instructions for use 


\title{
Passive Shimming by Eliminating Spherical Harmonics Coefficients of All Magnetic Field Components Generated by Correction Iron Pieces
}

\author{
So Noguchi ${ }^{1}$, SeokBeom $\mathrm{Kim}^{2}$, Seungyong $\mathrm{Hahn}^{3}$, and Yukikazu Iwasa ${ }^{3}$ \\ ${ }^{1}$ Graduate School of Information Science and Technology, Hokkaido University, Sapporo 060-0814, Japan \\ ${ }^{2}$ Graduate School of Natural Science and Technology, Okayama University, Okayama 700-8530, Japan \\ ${ }^{3}$ Francis Bitter Magnet Laboratory, Massachusetts Institute of Technology, Cambridge, MA 02139, USA
}

\begin{abstract}
When a magnet generating highly homogeneous magnetic field is designed, a shimming is required. The shimming is usually performed so that the magnetic field only in the axis direction is compensated by iron pieces and/or coils. The compensation is commonly achieved by eliminating the coefficients of the spherical harmonics expansion of the magnetic field generated by the main magnet. Some papers showed the coefficients of the spherical harmonics expansion in only the $z$-direction for passive shimming. However, recently some magnets generate a magnetic field tilted from the $z$-axis, such as a magic-angle-spinning NMR/MRI. It is, therefore, necessary to eliminate the coefficients of the spherical harmonics expansion in the $x$ - and $y$-directions.
\end{abstract}

Index Terms-Magnetic moment, MRI, NMR, passive shimming, spherical harmonics expansion.

\section{INTRODUCTION}

I $\mathrm{T}$ is necessary to compensate a magnetic field around the center with shimming when it is required to generate highly homogeneous magnetic field, such as MRI and NMR. The passive shimming, one of the shimming methods, is to compensate the magnetic field using some pieces of iron [1] [3]. Since the magnetic field homogeneity of a few PPM is required around the magnet center, the spherical harmonics expansion of the magnetic field is usually employed [4], [5].

A magnet for MRI or NMR is commonly axially symmetric. Therefore, only the $z$-component of the magnetic moment of correction iron pieces used for passive shimming is taken into account so that only the $z$-component of the magnetic field is homogenized [4]. The paper [5] presented the spherical harmonics coefficients of the $z$-component of the magnetic field generated by all the components of magnetic moment because the open MRI magnet generates the axially asymmetric magnetic field. In addition, the configuration of the correction iron pieces was optimized in order to eliminate the spherical harmonics coefficients.

Recently a newly developed magnet generates a magnetic field tilted from the $z$-axis for a magic-angle-spinning NMR [6]. Hence, the coefficients of the spherical harmonics expansion of all the magnetic field components, that are generated by all the components of the magnetic moment, are strongly desired to be eliminated by the passive shimming. Consequently, we derived the spherical harmonics coefficients of all the magnetic field components to all the magnetic moment components, and then we tried the passive shimming of the magic-angle-spinning NMR. In the passive shimming, the configuration of the correction iron pieces are optimized by the micro genetic algorithm $(\mu \mathrm{GA})$ in order to the spherical

Manuscript received June 29, 2013. Corresponding author: S. Noguchi (email: noguchi@ssi.ist.hokudai.ac.jp). harmonics coefficients of all the components.

\section{Coefficients of Spherical Harmonics}

\section{A. Spherical Harmonics Coefficients by Magnetic Moment}

The magnet flux $d \Phi$ generated by the magnetic moment $d \boldsymbol{m}$ at point $\mathrm{Q}$ as shown in Fig. 1(a) is presented by

$$
d \Phi=-\frac{d \boldsymbol{m}}{4 \pi \mu_{0}} \nabla_{Q}\left(\frac{1}{R}\right)
$$

where $\mu_{0}$ and $R$ are the permeability of free space and the distance between the points $\mathrm{Q}$ and $\mathrm{P}$ [7], respectively. Using the spherical harmonics function, $1 / R$ is given as

$$
\begin{aligned}
\frac{1}{R}= & \sum_{n=0}^{\infty} \sum_{m=0}^{n}\left\{\varepsilon_{m} \frac{(n-m) !}{(n+m) !} \frac{r^{n}}{r_{0}^{n+1}} P_{n}^{m}(\cos \alpha)\right. \\
& \left.\times P_{n}^{m}(\cos \theta) \cos [m(\psi-\phi)]\right\}
\end{aligned}
$$

where $\varepsilon_{m}$ is the Neumann factor, $P_{n}^{m}$ is the associated Legendre function, $r$ and $r_{0}$ are the distance from the origin to the points $\mathbf{P}$ and $\mathbf{Q}$, and $\alpha, \theta, \psi$, and $\phi$ are the angle shown in Fig. 1(a), respectively. The magnetic field $d \boldsymbol{B}_{\mathrm{m}}$ is expressed as

$$
d \boldsymbol{B}_{\mathrm{m}}=-\mu_{0} \nabla_{P} d \Phi .
$$

Here, the dimension of the correction iron piece with magnet moment $d \boldsymbol{m}=\left(d m_{\rho}, d m_{\phi}, d m_{z}\right)$ in cylindrical components is given in Fig. 1(b). The magnetic field $\boldsymbol{B}_{\mathrm{m}}$ generated by the correction iron piece is obtained by the integration of the volume, as follows:

$$
\boldsymbol{B}_{\mathrm{m}}=\int_{z_{1}}^{z_{2}} \int_{\rho_{1}}^{\rho_{2}} \int_{\psi_{1}}^{\psi_{2}} \frac{d \boldsymbol{m}}{4 \pi} \nabla_{P} \nabla_{Q}\left(\frac{1}{R}\right) \rho \mathrm{d} \psi \mathrm{d} \rho \mathrm{d} z .
$$

Here, it is assumed that the magnetic moment $d \boldsymbol{m}$ is constant in a correction iron piece. The following equation is derived 
from calculating (4).

$$
\begin{aligned}
\boldsymbol{B}_{\mathrm{m}}= & \sum_{n=0}^{\infty} \sum_{m=0}^{n}\left[r^{n} P_{n}^{m}(\cos \theta)\right. \\
& \left.\times\left\{\boldsymbol{C}^{\mathrm{s}}(n, m) \cos m \psi+\boldsymbol{D}^{\mathrm{s}}(n, m) \sin m \psi\right\}\right]
\end{aligned}
$$

where

$$
\begin{aligned}
{\left[\begin{array}{c}
\boldsymbol{C}^{\mathrm{s}}(n, m) \\
\boldsymbol{D}^{\mathrm{s}}(n, m)
\end{array}\right]=} & \boldsymbol{i}_{x}\left[\begin{array}{c}
C_{x}^{\mathrm{s}}(n, m) \\
D_{x}^{\mathrm{s}}(n, m)
\end{array}\right]+\boldsymbol{i}_{y}\left[\begin{array}{c}
C_{y}^{\mathrm{s}}(n, m) \\
D_{y}^{\mathrm{s}}(n, m)
\end{array}\right] \\
& +\boldsymbol{i}_{z}\left[\begin{array}{c}
C_{z}^{\mathrm{s}}(n, m) \\
D_{z}^{\mathrm{s}}(n, m)
\end{array}\right]
\end{aligned}
$$

and $\boldsymbol{i}_{x}, \boldsymbol{i}_{y}$, and $\boldsymbol{i}_{z}$ are the unit vector in the $x-, y$-, and $z$ direction, respectively. The detail of the coefficients $C^{s}$ and $D^{s}$ cannot be given here because of no space [8].

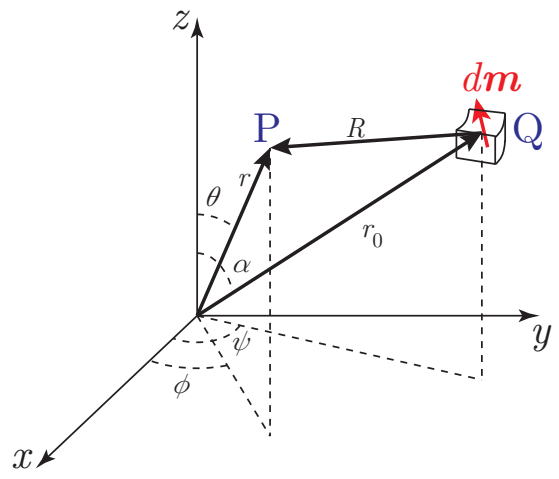

(a)

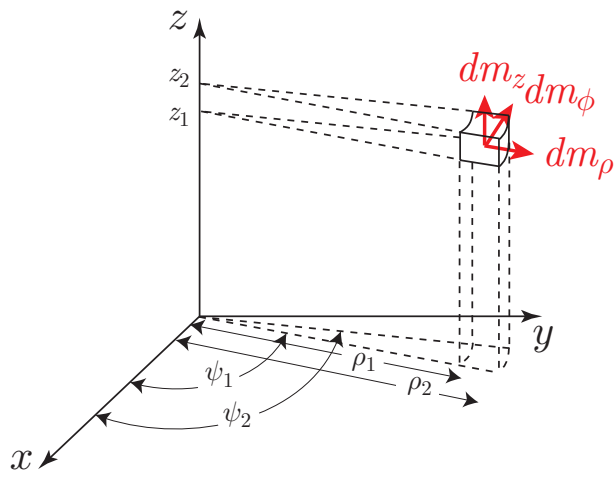

(b)

Fig. 1. (a) Magnetic moment $d \boldsymbol{m}$ at point $\mathrm{Q}$ generates magnetic flux $d \Phi$ at point $\mathrm{P}$ and (b) dimension of a piece of iron with $d \boldsymbol{m}=\left(d m_{\rho}, d m_{\phi}, d m_{z}\right)$ in the cylindrical components.

\section{B. Spherical Harmonics Coefficients by Current}

The current density $j$ with length of $d s$ generates the following magnetic vector potential $d \boldsymbol{A}$ :

$$
d \boldsymbol{A}=\mu_{0} \frac{j d s}{4 \pi R}
$$

where $R$ is the distance between the points $\mathrm{P}$ and $\mathrm{Q}$, replacing the magnetic moment $d \boldsymbol{m}$ with the current density element $j d s$ in Fig. 1(a). The following equation is derived from (2) and (7):

$$
\begin{aligned}
d \boldsymbol{A}= & \mu_{0} \frac{j d \boldsymbol{s}}{4 \pi r_{0}} \sum_{n=0}^{\infty} \sum_{m=0}^{n}\left\{\varepsilon_{m} \frac{(n-m) !}{(n+m) !} \frac{r^{n}}{r_{0}^{n}} P_{n}^{m}(\cos \alpha)\right. \\
& \left.\times P_{n}^{m}(\cos \theta) \cos [m(\psi-\phi)]\right\}
\end{aligned}
$$

The magnetic field generated by the current $\boldsymbol{B}_{\mathrm{c}}$ is

$$
\boldsymbol{B}_{\mathrm{c}}=\int_{v} \nabla \times d \boldsymbol{A} \mathrm{d} v
$$

where $v$ is the volume of the magnet. Finally, we can obtain using (7) - (9)

$$
\begin{aligned}
\boldsymbol{B}_{\mathrm{c}}= & \sum_{n=0}^{\infty} \sum_{m=0}^{n}\left[r^{n} P_{n}^{m}(\cos \theta)\right. \\
& \left.\times\left\{\boldsymbol{C}^{\mathrm{c}}(n, m) \cos m \psi+\boldsymbol{D}^{\mathrm{c}}(n, m) \sin m \psi\right\}\right](10)
\end{aligned}
$$

where

$$
\begin{aligned}
{\left[\begin{array}{c}
\boldsymbol{C}^{\mathrm{c}}(n, m) \\
\boldsymbol{D}^{\mathrm{c}}(n, m)
\end{array}\right]=} & \boldsymbol{i}_{x}\left[\begin{array}{c}
C_{x}^{\mathrm{c}}(n, m) \\
D_{x}^{\mathrm{c}}(n, m)
\end{array}\right]+\boldsymbol{i}_{y}\left[\begin{array}{c}
C_{y}^{\mathrm{c}}(n, m) \\
D_{y}^{\mathrm{c}}(n, m)
\end{array}\right] \\
& +\boldsymbol{i}_{z}\left[\begin{array}{c}
C_{z}^{\mathrm{c}}(n, m) \\
D_{z}^{\mathrm{c}}(n, m)
\end{array}\right] .
\end{aligned}
$$

The coefficients $C^{c}$ and $D^{c}$ of the ring coil are represented in [7]. It is, however, necessary to compute the coefficients $C^{c}$ and $D^{c}$ by a numerical integration when the magnet configuration is axially asymmetric, such as a dipole magnet.

When the main magnet generates a tilted magnetic field, the spherical harmonics coefficients can be transformed using the method presented in [9].

\section{Passive Shimming}

For compensating the magnetic field with passive shimming,

$$
\left[\begin{array}{l}
\boldsymbol{C}^{\mathrm{s}}(n, m) \\
\boldsymbol{D}^{\mathrm{s}}(n, m)
\end{array}\right]+\left[\begin{array}{l}
\boldsymbol{C}^{\mathrm{c}}(n, m) \\
\boldsymbol{D}^{\mathrm{c}}(n, m)
\end{array}\right]=\left[\begin{array}{l}
0 \\
0
\end{array}\right]
$$

with $n \geq 1$ has to be satisfied.

In the passive shimming, the configuration of the correction iron pieces has to be optimized in order to eliminate the coefficients $\boldsymbol{C}^{\mathrm{c}}(n, m)$ and $\boldsymbol{D}^{\mathrm{c}}(n, m)$ by adding $\boldsymbol{D}^{\mathrm{s}}(n, m)$ and $\boldsymbol{D}^{\mathrm{s}}(n, m)$. The magnetic moment $d \boldsymbol{m}$ is computed by a numerical simulation method, such as a finite element method or a magnetic moment method.

\section{PAssive ShIMMING}

\section{A. Magic-Angle-Spinning NMR}

The passive shimming is applied to a pair of dipole magnets of the magic-angle-spinning NMR [6]. Fig. 2 shows the dipole magnets with the tilt of $54.74 \mathrm{deg}$. in the $y$ - $z$ plane, the center magnetic field is $0.3 \mathrm{~T}$, and its dimensions are referred in [6]. Table I shows the spherical harmonics coefficients of the magnetic field generated by the dipole magnets, however $n \leq$ 4.

For passive shimming, the $\mu \mathrm{GA}[10]$ optimizes the configuration of the correction iron pieces. Considering the symmetry, the design region of the correction iron pieces, as shown in Fig. 2, is divided into 2 (radially) $\times 36$ (circumferentially) 


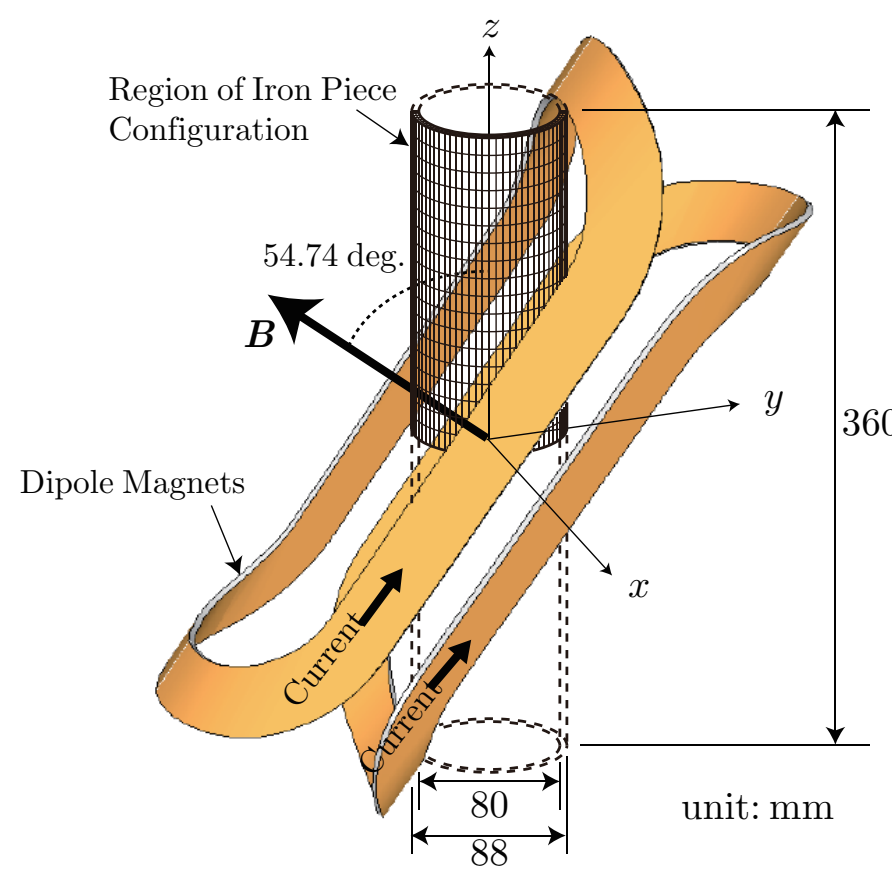

Fig. 2. Dipole magnets for magic-angle-spinning NMR. The configuration of the iron piece is optimized by the $\mu \mathrm{GA}$. The quarter region of the configuration is considered due to the symmetry, and it is divided into 2 (radially) $\times 36$ (circumferentially) $\times 18$ (heigtwise) segmental elements.

TABLE I

Spherical Harmonics CoefFicients of Dipole Magnets

\begin{tabular}{lrrr}
\hline Coefficient & $x$ component & $y$ component & $z$ component \\
\hline$C^{\mathrm{c}}(0,0)$ & 0.0 & -0.24444 & 0.17281 \\
$C^{\mathrm{c}}(1,0)$ & 0.0 & 0.0 & 0.0 \\
$C^{\mathrm{c}}(1,1)$ & 0.0 & 0.0 & 0.0 \\
$C^{\mathrm{c}}(2,0)$ & 0.0 & -0.74069 & 1.06713 \\
$C^{\mathrm{c}}(2,1)$ & -0.86867 & 0.0 & 0.0 \\
$C^{\mathrm{c}}(2,2)$ & 0.0 & 0.45035 & -0.26435 \\
$C^{\mathrm{c}}(3,0)$ & 0.0 & 0.0 & 0.0 \\
$C^{\mathrm{c}}(3,1)$ & 0.0 & 0.0 & 0.0 \\
$C^{\mathrm{c}}(3,2)$ & 0.0 & 0.0 & 0.0 \\
$C^{\mathrm{c}}(3,3)$ & 0.0 & 0.0 & 0.0 \\
$C^{\mathrm{c}}(4,0)$ & 0.0 & 1004.881 & -70.779 \\
$C^{\mathrm{c}}(4,1)$ & 296.141 & 0.0 & 0.0 \\
$C^{\mathrm{c}}(4,2)$ & 0.0 & -82.389 & 58.331 \\
$C^{\mathrm{c}}(4,3)$ & -63.652 & 0.0 & 0.0 \\
$C^{\mathrm{c}}(4,4)$ & 0.0 & 18.288 & -12.930 \\
$D^{\mathrm{c}}(1,1)$ & 0.0 & 0.0 & 0.0 \\
$D^{\mathrm{c}}(2,1)$ & 0.0 & 0.14871 & -0.19576 \\
$D^{\mathrm{c}}(2,2)$ & 0.61434 & 0.0 & 0.0 \\
$D^{\mathrm{c}}(3,1)$ & 0.0 & 0.0 & 0.0 \\
$D^{\mathrm{c}}(3,2)$ & 0.0 & 0.0 & 0.0 \\
$D^{\mathrm{c}}(3,3)$ & 0.0 & 0.0 & 0.0 \\
$D^{\mathrm{c}}(4,1)$ & 0.0 & -27.0566 & 0.0 \\
$D^{\mathrm{c}}(4,2)$ & -140.003 & 0.0 & -0.19576 \\
$D^{\mathrm{c}}(4,3)$ & 0.0 & 39.791 & 0.0 \\
$D^{\mathrm{c}}(4,4)$ & 25.005 & 0.0 & 0.0 \\
\hline
\end{tabular}

$\times 18$ (heightwise) segmental elements, and the $\mu \mathrm{GA}$ decides whether the iron piece exists in a subdivision or not, like a topology optimization [11]. The object function $F$ to be minimize is

$$
F=\sum_{i=x, y, z} \sum_{n=1}^{4} \sum_{m=0}^{n} w(n)\left(\left|C_{i}^{\mathrm{s}}(n, m)+C_{i}^{\mathrm{c}}(n, m)\right|\right.
$$

$$
\left.+\left|D_{i}^{\mathrm{s}}(n, m)+D_{i}^{\mathrm{c}}(n, m)\right|\right) .
$$

where $w(n)$ is the following weight function.

$$
w(n)=10^{-2 n}
$$

The weight function is decided according to the desired bore size of the homogenized magnetic field. When whole the iron pieces are absent, the initial objective function $F=4.634 \times$ $10^{-4}$.

The magnetic moment $d \boldsymbol{m}$ of every iron piece is computed by the magnetic moment method in the cylindrical coordinate.

\section{B. Passive Shimming Result}

Fig. 3 shows the optimized configuration of the correction iron pieces, and Table II represents the spherical harmonics coefficients of the magnetic field affected by the correction iron pieces. The objective function optimized is $F=0.806 \times$ $10^{-4}$. Figs. 4 and 5 shows the absolute value of the nonzero spherical harmonics coefficients with $n=2$ and 4 , respectively. The coefficients of $n=2$ are reduced adequately, however, those of $n=4$ are not.

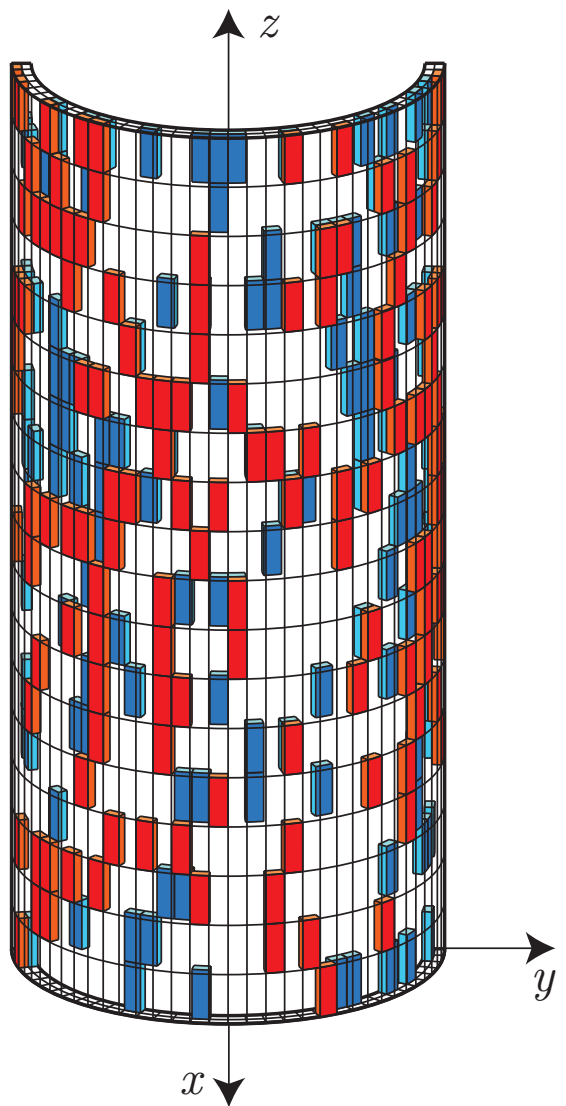

Fig. 3. The optimized configuration of correction iron pieces by $\mu \mathrm{GA}$. The blue-colored pieces are in the inner layer, and the red-colored ones in the outer.

Table III shows the inhomogeneity of the magnetic field within $10 \mathrm{~mm}$ sphere to the dipole magnets with and without passive shimming. The inhomogeneity is drastically improved to $4.3 \mathrm{ppm}$. In this optimization, the size of the iron piece is too large to reduce all the spherical harmonics coefficients largely. 
TABLE II

SPHERICAL HARMONICS COEFFICIENTS OF IRON PIECES

\begin{tabular}{lrrr}
\hline Coefficient & $x$ component & $y$ component & $z$ component \\
\hline$C^{\mathrm{s}}(0,0)$ & 0.0 & -0.00367 & 0.00217 \\
$C^{\mathrm{s}}(1,0)$ & 0.0 & 0.0 & 0.0 \\
$C^{\mathrm{s}}(1,1)$ & 0.0 & 0.0 & 0.0 \\
$C^{\mathrm{s}}(2,0)$ & 0.0 & 0.65458 & -1.06536 \\
$C^{\mathrm{s}}(2,1)$ & 0.86747 & 0.0 & 0.0 \\
$C^{\mathrm{s}}(2,2)$ & 0.0 & -0.45050 & 0.25618 \\
$C^{\mathrm{s}}(3,0)$ & 0.0 & 0.0 & 0.0 \\
$C^{\mathrm{s}}(3,1)$ & 0.0 & 0.0 & 0.0 \\
$C^{\mathrm{s}}(3,2)$ & 0.0 & 0.0 & 0.0 \\
$C^{\mathrm{s}}(3,3)$ & 0.0 & 0.0 & 0.0 \\
$C^{\mathrm{s}}(4,0)$ & 0.0 & -652.938 & -150.717 \\
$C^{\mathrm{s}}(4,1)$ & -221.509 & 0.0 & 0.0 \\
$C^{\mathrm{s}}(4,2)$ & 0.0 & 16.939 & -83.884 \\
$C^{\mathrm{s}}(4,3)$ & 33.197 & 0.0 & 0.0 \\
$C^{\mathrm{s}}(4,4)$ & 0.0 & 10.404 & 5.949 \\
$D^{\mathrm{s}}(1,1)$ & 0.0 & 0.0 & 0.0 \\
$D^{\mathrm{s}}(2,1)$ & 0.0 & -0.13893 & 0.43639 \\
$D^{\mathrm{s}}(2,2)$ & -0.93808 & 0.0 & 0.0 \\
$D^{\mathrm{s}}(3,1)$ & 0.0 & 0.0 & 0.0 \\
$D^{\mathrm{s}}(3,2)$ & 0.0 & 0.0 & 0.0 \\
$D^{\mathrm{s}}(3,3)$ & 0.0 & 0.0 & 0.0 \\
$D^{\mathrm{s}}(4,1)$ & 0.0 & 255.983 & -261.175 \\
$D^{\mathrm{s}}(4,2)$ & 25.019 & 0.0 & 0.0 \\
$D^{\mathrm{s}}(4,3)$ & 0.0 & -21.214 & 8.166 \\
$D^{\mathrm{s}}(4,4)$ & 6.058 & 0.0 & 0.0 \\
\hline
\end{tabular}

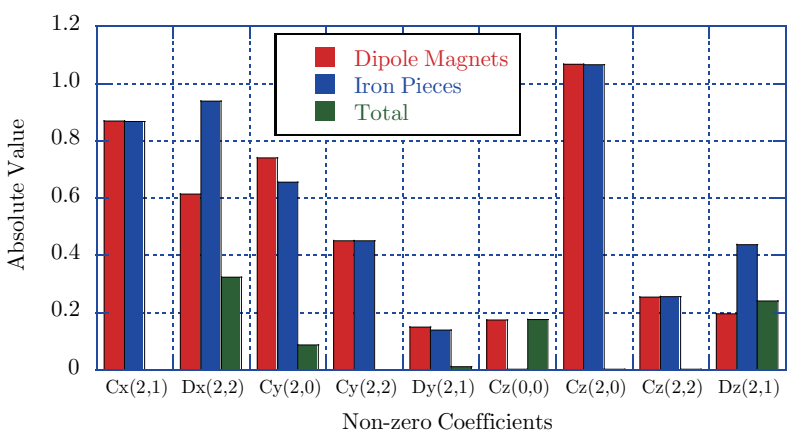

Fig. 4. Absolute value of the non-zero spherical harmonics coefficients with $n=2$.

However, it was successful to compute the spherical harmonics coefficients of all the component by all the magnetic moment components, and the optimization of the correction iron piece configuration was done.

TABLE III

InHomogeneity of MAgnetic Field Within 10 MM Sphere

\begin{tabular}{lrr}
\hline & w/o shimming (ppm) & with shimming (ppm) \\
\hline$x$-component & 0.0 & 0.0 \\
$y$-component & 137.7 & 3.9 \\
$z$-component & 104.0 & 1.8 \\
All components & 172.5 & 4.3 \\
\hline
\end{tabular}

\section{CONCLUSION}

We calculate the spherical harmonics coefficients of all the components generated by all the magnetic moment components. When a main magnet is tilted, it is necessary to eliminate the spherical harmonics of all the components by passive shimming. We have tried to compensate the magnetic

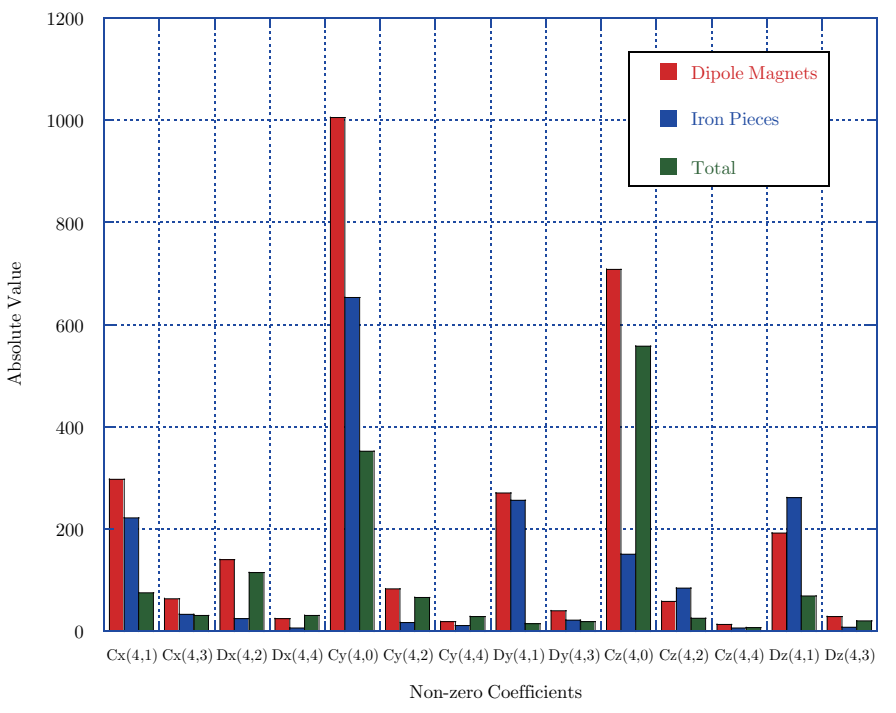

Fig. 5. Absolute value of the non-zero spherical harmonics coefficients with $n=4$.

field of the tilted dipole magnets so that the configuration of the correction iron pieces was optimized by the $\mu \mathrm{GA}$. In the future, it is necessary to eliminate higher order coefficients with a smaller piece of iron. In addition, it is necessary to employ active shimming with passive one. The proposed method in this paper is useful in the simultaneous active and passive shimming.

\section{REFERENCES}

[1] A. Belov, V. Bushuev, M. Emelianov, V. Emelianov, V. Eregin, Y. Severgin, S. Sytchevski, and V. Vasiliev, "Passive shimming of the superconducting magnet for MRI," IEEE Trans. Appl. Supercond., vol. 5, no. 2, pp. 679-681, 1995.

[2] L. Feng, Z. Jianfeng, X. Lin, and S. Crozier, "A hybrid feild-harmonics approach for passive shimming design in MRI," IEEE Trans. Appl. Supercond., vol. 21, no. 2, pp. 60-67, Apr. 2011.

[3] Y. Zhang, D. Xie, B. Bai, H. S. Yoon, and C. S. Koh, "A novel optimal design method of passive shimming for permanent MRI magnet," IEEE Trans. Magn., vol. 44, no. 6, pp. 1058-1061, Jun. 2008.

[4] H. S. Lopez, F. Liu, W. Weber, and S. Crozier, "Passive shim and a shimming approach for biplanar permanent magnetic resonance imaging magnets," IEEE Trans. Magn., vol. 44, no. 3, pp. 394-402, Mar. 2008.

[5] S. Kakugawa, H. Hino, A. Komura, M. Kiatmura, H. Takeshima, T. Yatsuo, and H. Tazaki, "Three-dimensional optimization of correction iron pieces for open high field MRI system," IEEE Trans. Appl. Supercond., vol. 14, no. 2, pp. 1624-1627, Jun. 2004.

[6] J. Voccio, S. Hahn, D. K. Park, J. Ling, Y. Kim, J. Bascuñán, and Y. Iwasa, "Magic-angle-spinning NMR magnet development: field analysis and prototype," IEEE Trans. Appl. Supercond., vol. 23, no. 3, p. 4300804, Jun. 2013.

[7] F. Roméo and D. I. Hoult, "Magnet field profiling: analysis and correcting coil design," Magnetic Resonance in Medicine, vol. 1, pp. 44-65, 1984.

[8] S. Noguchi, F. Nobuyama, S. B. Kim, S. Hahn, and Y. Iwasa, "Spherical harmonics coefficients of all magnetic field components generated by iron piece," Proc. 19th COMPUMAG, PB5-14, Jul. 2013.

[9] M. A. Blanco, M. Flórez, and M. Bermejo, "Evaluation of the rotation matrices in the basis of real spherical harmonics," Journal of Molecular Structure, vol. 419, pp. 19-27, 1997.

[10] Y. Watanabe, K. Watanabe, and H. Igarashi, "Optimization of meander line antenna considering coupling between nonlinear circuit and electromagnetic waves for UHF-band RFID," IEEE Trans. Magn., vol. 47, no. 5, pp. 1506-1509, May 2011.

[11] Y. Tominaga, Y. Okamoto, S. Wakao, and S. Sato, "Binary-based topology optimization of magnetostatic shielding by a hybrid evolutionary algorithm combining genetic algorithm and extended compact genetic algorithm," IEEE Trans. Magn., vol. 49, no. 5, pp. 2093-2096, May 2013. 